\title{
Crioterapia moderada: uma alternativa no tratamento da lesão celular induzida pelo exercício
}

\section{Moderate cryotherapy by immersion: an alternative in the treatment of muscle damage induced by exercise}

\author{
Manoel da Cunha Costa ${ }^{1,2}$, Raphael José Perrier-Melo ${ }^{1,2}$, Jorge Luiz de \\ Brito-Gomes $^{1,2}$, Tetsuo Tashiro ${ }^{3}$, José Manuel Costa Soares ${ }^{4}$
}

http://dx.doi.org/10.11606/issn.2238-6149.v28i2p239-245

Costa MC, Perrier-Melo RJ, Brito-Gomes JL, Tashiro T, Soares JMC. Crioterapia moderada: uma alternativa no tratamento da lesão celular induzida pelo exercício. Rev Ter Ocup Univ São Paulo. 2017 maio-ago.;28(2):239-45.

RESUMO: Objetivo: analisar o efeito da crioterapia moderada com imersão $\left(15^{\circ}\right.$ celsius - C), nas respostas inflamatórias musculares, nas variáveis bioquímicas e neuromotoras após uma sessão de exercício de força excêntrico. Procedimentos Metodológicos: 18 homens (18-25 anos) saudáveis, não praticantes de exercício físico e que participaram voluntariamente da pesquisa foram recrutados. Inicialmente foram randomizados em três grupos: Grupo Controle (GC), Grupo Exercício (GE) e Grupo Exercício + Crioterapia (EX $+\mathrm{CT}$ ). Após a aleatorização, os voluntários seguiram para realização de testes: a) bioquímico (Creatina Quinase - CK), b) neuromotor (amplitude de movimento - ÂNGULO), c) escala de dor - DOR e d) um teste de força isométrica máxima. Em seguida, os grupos experimentais realizaram séries de ações excêntricas máximas para região anterior e posterior da perna no exercício sentadinha. Após a sessão analisou-se CK, ÂNGULO e DOR, nos momentos 24, 48, 72,96 e 120 horas. Os sujeitos do EX + CT receberam tratamento crioterápico imediatamente após a sessão e nos momentos 12, 24, 36 e 48 horas subsequentes. Resultados: Em comparação com o repouso, os valores de CK, DOR e ÂNGULO apresentaram aumento significativo após as sessões nos grupos experimentais. O GE exibiu valores significativamente superior ao $\mathrm{EX}+\mathrm{CT}$ em todas as variáveis analisadas. Conclusão: A crioterapia mostrou-se uma alternativa viável no tratamento da miopatia do exercício e da dor muscular tardia.

DESCRITORES: Crioterapia; Exercício, Analgesia.
Costa MC, Perrier-Melo RJ, Brito-Gomes JL, Tashiro T, Soares JMC. Moderate cryotherapy by immersion: an alternative in the treatment of muscle damage induced by exercise. Rev Ter Ocup Univ São Paulo. 2017 May-Aug.;28(2):239-45.

ABSTRACT: Objective: to analyze the effect of moderate immersion cryotherapy $\left(15^{\circ} \mathrm{C}\right)$ in muscular inflammatory responses, in biochemical and neuromotor variables, after a session of eccentric exercise. Methods: 18 healthy men (aged 1825 years), non-practitioners of physical exercise who participated in the survey on a voluntary basis were recruited. Initially, they were randomized into three groups: Control group (CG), Exercise Group (EX) and Exercise + Cryotherapy Group (EX + CT). After randomization, volunteers took: a) biochemical test (creatine kinase-CK), b) neuromotor test (range of motion - ANGLE), c) test of pain scale - PAIN, and d) maximum isometric strength test. Then, the experimental groups performed a series of maximal eccentric actions (seated calf raise) for the anterior and posterior region of the leg. CK, ANGLE and PAIN were analyzed 24, 48, 72, 96 and 120 hours after the session. The subjects on EX + CT received cryotherapy treatment immediately after the session and 12, 24 and, 36 hours thereafter. Results: In comparison to resting the CK, PAIN and ANGLE values showed significant increase after the sessions in the experimental groups. The EX exhibited significantly higher values than those of EX $+\mathrm{CT}$ in all variables analyzed. Conclusion: Cryotherapy indicate to be a viable alternative in the treatment for myopathy and delayed onset muscle soreness.

KEYWORDS: Cryotherapy; Exercise; Analgesia.

Parte da tese de doutorado "Crioterapia: Efeitos na Homeostasia Muscular Após o Exercício", de Manoel da Cunha Costa, Porto, Portugal. 1. Universidade de Pernambuco, Escola Superior de Educação Física, Recife, PE, Brasil.

2. Grupo de Pesquisa em Avaliação da Performance Humana, Recife, PE, Brasil. E-mail: mcosta2@gmail.com, perrierprof@gmail.com, jorgelbritog@hotmail.com

3. Universidade Federal de Pernambuco, Departamento de Educação Física, Recife, PE, Brasil. E-mail: tedbio@oi.com.br

4. Universidade do Porto, Faculdade de Desporto, Porto, Portugal. E-mail: jmsoares@fade.up.pt

Endereço para correspondência: Rua Arnóbio de Marques, 310. Santo Amaro, Recife, Pernambuco, Brasil. CEP: 50100-130. 


\section{INTRODUÇÃO}

$\mathrm{D}$ urante a realização de exercícios físicos ocorrem contrações musculares isotônicas ou isométricas, havendo uma quebra na homeostasia, que promove de maneira progressiva o desenvolvimento de estímulos metabólicos no tecido muscular esquelético, acarretando na criação e/ou propagação de micro lesões induzidas pelo exercício físico, gerando uma sensação de desconforto na musculatura esquelética, podendo durar até 72 horas ${ }^{1}$. Assim, sabe-se que durante a ação muscular excêntrica, a dor muscular ocorre em maior magnitude, devido a maior proliferação de metabólitos².

As lesões nas fibras musculares geralmente acontecem de forma transitória, quando há um elevado nível de estresse, caracterizado pela degradação ou danificação das células do tecido muscular. Esse processo inflamatório intensifica de maneira significativa o dano muscular, causando alterações na sua estrutura, assim como na sua função, provocando aumentos na concentração de creatina quinase (CK) circulante no meio extra celular ${ }^{3}$.

Existem diversas alternativas para minimizar o processo da dor muscular tardia (DMT), e uma delas é a crioterapia. Esta técnica, de custo reduzido e fácil aplicação, é caracterizada por ser uma terapia com aplicação de baixas temperaturas na área alvo, capaz de promover analgesia na região afetada e diminuição da temperatura local, favorecendo ajustes fisiológicos ${ }^{4-6}$, redução de transmissão nervosa e do metabolismo celular, que minimiza os processos inflamatórios, com consequente diminuição do desconforto muscular; redução na formação de edema e danos teciduais ${ }^{5,7}$.

$\mathrm{Na}$ literatura encontra-se diversas propostas para aplicação do tratamento crioterápico, entre elas, as mais comuns são os sprays, compressas, bolsas térmicas, imersão em água com baixas temperaturas e aplicação direta com gelo ${ }^{8}$. As técnicas, variam de acordo com a duração da aplicação (um minuto e 40 segundos à 25 minutos) e temperatura estabelecida ( $0^{\circ}$ à $29^{\circ}$ Celsius), todas com objetivos de reduzir o estresse muscular, diminuindo os riscos do agravamento da lesão ósteo-mio-articular ${ }^{8,9}$.

Entre os métodos de tratamento crioterápico, a técnica de imersão é a única capaz de gerar maior contato da área muscular lesada com o ambiente resfriado, proporcionando controle na temperatura e capacidade de reduzir a agressão tecidual ${ }^{10}$. Contudo, mesmo sendo uma técnica muito utilizada em programas de reabilitação articular, muscular, óssea e na melhoria de desempenho, ainda não há consenso a respeito do tempo e temperatura ideal, necessários para promover benefícios terapêuticos do tratamento com crioterapia em humanos ${ }^{8}$. A hipótese da pesquisa é que a crioterapia moderada (técnica de imersão) à $15^{\circ} \mathrm{C}$ é capaz de causar benefícios sobre aspectos bioquímicos e na mobilidade articular em humanos. Sendo assim, o objetivo do estudo foi de analisar o efeito da crioterapia moderada com imersão a $15^{\circ} \mathrm{C}$, nas respostas inflamatórias musculares em humanos nas variáveis bioquímicas e neuromotoras após uma sessão de exercício de força excêntrico.

\section{PROCEDIMENTOS METODOLÓGICOS}

O presente manuscrito é fruto da primeira parte de uma tese de doutorado defendida em 2002. Trata-se de uma estudo do tipo quase-experimental e quantitativo, com amostra aleatória, realizado na cidade de Recife, PE, Brasil. Aprovado pelo Comitê de Ética em Pesquisa da Universidade de Pernambuco, sob o protocolo: 0125.

\section{Amostra}

A amostra foi constituída por 18 sujeitos adultos saudáveis (23,2 $\pm 2,3$ anos), universitários do gênero masculino, não praticantes de exercício físico e sem fatores de risco (avaliado pelo Questionário de Prontidão para Atividade Física - PAR-Q), que participaram voluntariamente da pesquisa. Foram incluídos: os que não apresentavam histórico de lesão/dor/desconforto sobre a musculatura do membro inferior e não realizavam tratamento farmacológico atual com uso de analgésicos. Foram excluídos os que apresentassem alguma lesão que impedisse a realização do procedimento experimental e não finalizassem uma das etapas do estudo, ou iniciassem qualquer tipo de exercício físico durante o período de coleta.

\section{Delineamento experimental}

\section{Primeira etapa}

Inicialmente os sujeitos receberam informações gerais a respeito do estudo, dos procedimentos a serem adotados nas coletas, e os que responderam negativamente ao PAR-Q, foram orientados a assinar o Termo de Consentimento Livre e Esclarecido. Em seguida foram divididos de forma aleatória em três grupos: Grupo Controle (CON) n=6, Grupo de Exercício Excêntrico (GE) n $=6$ e Grupo Exercício Excêntrico + Crioterapia $(E X+C T)$ 
$\mathrm{n}=6$. Após a aleatorização dos grupos, seguiram para realização de uma bateria de testes:

\section{Bioquímico (agressão tecidual CK)}

Para determinação da concentração enzimática de CK no plasma, foram coletados em punção digital, $32 \mu \mathrm{L}$ de sangue capilar dos sujeitos, após limpeza do local com algodão embebido em álcool $70 \%$. Em seguida, foi utilizado uma lanceta com disparador automático e o sangue foi drenado para um tubo previamente heparinizado, e adicionado à uma fita reativa de $\mathrm{CK}$ e colocado no aparelho Reflotron (Boehringer Roche Mannheim), para análise imediata.

\section{Neuromotor (amplitude de movimento)}

A amplitude de movimento foi determinada pela diferença no ângulo do tornozelo entre o somatório das flexões plantar e dorsal, verificada por meio de um flexímetro (Code Research Institute), colocado no dorso do pé, com o sujeito sentado com o joelho fletido à $90^{\circ}$. Cada ângulo foi mensurado três vezes, considerando o maior valor, expresso em graus.

\section{Percepção subjetiva de esforço (escala de dor-sem palpação)}

A escala de dor foi medida por meio de uma escala analógica numérica de dor (1-7) adaptada por Clarkson e Hubal $^{10}$, na qual "um" indica ausência de dor (sem dor), e "sete" é considerado o máximo de dor (dor insuportável). A determinação do valor na escala foi realizada através de uma resposta verbal, no qual o sujeito indicava um número na escala, referente à percepção de dor sem palpação.

\section{Teste de Força Isométrica Máxima (TFIM)}

Consistiu em medir a capacidade de realizar a força isométrica máxima por meio de uma célula de carga (Globus-ergo meter - Italy). Assim, posteriormente foi utilizado o percentual do resultado do teste no protocolo experimental para indução de danos musculares a partir do exercício de força excêntrico.

\section{Segunda etapa}

Caracterizada pela realização de exercício de força excêntrico por parte dos grupos experimentais, como forma de indução aos danos musculares. $\mathrm{O}$ grupo controle não realizou exercícios durante o período do estudo, mantendo as atividades diárias normais.

\section{Exercício de força excêntrico}

A sessão experimental consistiu na realização de exercícios dinâmicos com ênfase na fase excêntrica. Durante a fase concêntrica os avaliadores levantavam a carga, a fim de evitar trabalho muscular nessa etapa, para que o avaliado suportasse apenas a fase excêntrica do movimento. Todos voluntários realizaram séries de cinco ações excêntricas com intervalo de 30 segundos, para o trabalho da musculatura da perna (músculo sóleo), por meio do equipamento Panturrilheira (Nippon), sendo aplicado uma intensidade de $85 \%$ da força isométrica máxima.

Cada voluntário foi posicionado na cadeira na posição sentada, com os cotovelos fletidos à $90^{\circ}$ e mãos no suporte da panturrilheira para maior acomodação do sujeito ao instrumento utilizado para coleta. Além disso, a altura do banco foi ajustada de forma que os joelhos permanecessem à $90^{\circ}$, e os pés posicionados na base da panturrilheira no qual foi realizado a força isométrica máxima. O GE realizou uma sessão de exercício e o $\mathrm{EX}+\mathrm{CT}$ realizou uma sessão de exercício, em seguida as sessões de crioterapia.

\section{Crioterapia}

O tratamento com crioterapia moderada, foi realizado com a utilização de um tanque plástico (340 litros), com a passagem da água em circuito aberto, adaptado por meio de um equipamento de congelador (Brastemp), mantendo uma temperatura de $15^{\circ} \mathrm{C}$, aceitando-se uma variação de $\pm 1^{\circ}$ Celsius $(\mathrm{C})$, monitorada constantemente durante todo o processo de crioterapia por dois termômetros da marca Incoterm $^{\circledR}$. Os sujeitos foram mantidos em posição sentada ao lado do tanque plástico, imergindo a parte distal da perna (do hálux até a patela) na água com a temperatura controlada à $15^{\circ} \mathrm{C}$. A aplicação do frio como elemento terapêutico foi realizada da seguinte maneira: quatro sessões de imersão à água, com intervalos de doze horas entre elas, a uma temperatura de $15^{\circ} \mathrm{C}$ no tanque plástico, durante trinta minutos de acordo com a Figura 1.

Os grupos refizeram as baterias de testes após um período de $24 \mathrm{~h}, 48 \mathrm{~h}, 72 \mathrm{~h}, 96$ horas e 1 semana, conforme demonstrado na Figura 1. Neste sentido, para não alterar os valores referente às variáveis analisadas, os sujeitos foram orientados a não realizar exercícios físicos durante o período de avaliação. 


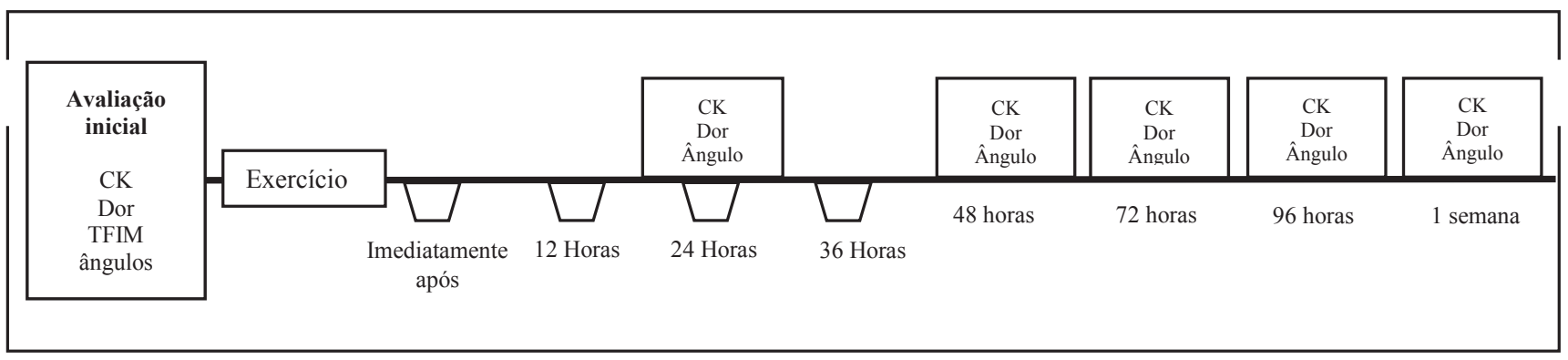

Legenda: Exercício - sessão experimental; CK - análise bioquímica da creatina quinase; DOR - avaliação da escala de dor; TFIM- Teste de Força Isométrica Máxima; ÂNGULOS - análise da amplitude do movimento; CRIOTERAPIA - sessão de trinta minutos com imersão de perna em um reservatório à $15^{\circ} \mathrm{C}$.

Figura 1 - Desenho do estudo

\section{Procedimentos estatísticos}

Foi realizada uma análise de normalidade e homogeneidade por meio do teste de Shapiro-Wilk e Levene, respectivamente. Os dados estão apresentados em média e desvio padrão. Para verificar as diferenças entre os momentos de avaliação, foi realizado o teste Anova de medidas repetidas, e para comprovação das diferenças entre as médias nos diferentes momentos foi utilizado o teste de Post Hockey de Tuckey, adotando um nível de significância de $p<0,05$.

\section{RESULTADOS}

$\mathrm{Na}$ Tabela 1 são apresentadas as respostas da variável bioquímica (creatina quinase-CK), neuromotora (variação da amplitude do movimento - ÂNGULO) e escala de percepção da dor (DOR) durante as fases de avaliação.

As variáveis CK, dor e ângulo da articulação foram medidas em seis momentos. No presente estudo os níveis de $\mathrm{CK}$ foram medidos do repouso às 120 horas, com intervalo de 24 horas entre as medidas. As concentrações plasmáticas de CK durante os tempos de avaliação, comparadas com o repouso apresentaram maiores valores após as sessões exercícios (EX e EX+CT). Contudo, o grupo EX exibiu maiores valores, com diferenças significativas em 24 e 48 horas, com reduções após 72 horas. Já no grupo $\mathrm{EX}+\mathrm{CT}$ foi verificado aumento significativo apenas no momento 24 horas, com reduções a partir das 48 horas. No grupo controle, não foram encontradas diferenças significativas.

Tabela 1 - Comportamento da CK, DOR e ÂNGULO entre os grupos, apresentados em média \pm desvio-padrão

\begin{tabular}{|c|c|c|c|c|c|c|}
\hline & REPOUSO & $24 \mathrm{~h}$ & $48 \mathrm{~h}$ & $72 \mathrm{~h}$ & $96 \mathrm{~h}$ & 1 Semana \\
\hline \multicolumn{7}{|c|}{$\operatorname{COM}(n=6)$} \\
\hline CK (U/L) & $44,3 \pm 13,4$ & $44,0 \pm 13,9$ & $44,6 \pm 14,0$ & $45,0 \pm 14,7$ & $44,2 \pm 13,7$ & $44,7 \pm 13,7$ \\
\hline Dor & $1,00 \pm 0,00$ & $1,00 \pm 0,00$ & $1,00 \pm 0,00$ & $1,00 \pm 0,00$ & $1,00 \pm 0,00$ & $1,00 \pm 0,00$ \\
\hline Ângulo $\left({ }^{\circ}\right)$ & $92,2 \pm 3,7$ & $92,0 \pm 3,7$ & $92,5 \pm 3,5$ & $92,0 \pm 3,4$ & $92,5 \pm 4,0$ & $92,5 \pm 3,8$ \\
\hline \multicolumn{7}{|l|}{$E X(n=6)$} \\
\hline CK (U/L) & $42,1 \pm 20,5$ & $241,4 \pm 128,2 *$ & $178,7 \pm 106,4^{*}$ & $113,5 \pm 52,0$ & $77,1 \pm 33,7$ & $48,1 \pm 20,9$ \\
\hline Dor & $1,00 \pm 0,00$ & $3,00 \pm 1,26 *$ & $2,70 \pm 1,97^{*}$ & $2,30 \pm 1,97^{*}$ & $1,70 \pm 0,82 *$ & $1,20 \pm 0,41$ \\
\hline Ângulo $\left({ }^{\circ}\right)$ & $87,7 \pm 4,9$ & $79,2 \pm 4,8^{*}$ & $72,5 \pm 9,8^{*}$ & $78,7 \pm 9,5^{*}$ & $81,8 \pm 5,7 *$ & $86,5 \pm 3,8$ \\
\hline \multicolumn{7}{|c|}{$E X+C T(n=6)$} \\
\hline $\mathrm{CK}(\mathrm{U} / \mathrm{L})$ & $42,8 \pm 27,0$ & $196,9 \pm 98,6^{*}$ & $129,8 \pm 70,5$ & $102,0 \pm 57,9$ & $74,1 \pm 48,5$ & $49,0 \pm 25,5$ \\
\hline Dor & $1,00 \pm 0,00$ & $2,00 \pm 0,89^{*}$ & $2,20 \pm 0,98^{*}$ & $1,20 \pm 0,41$ & $1,00 \pm 0,00$ & $1,00 \pm 0,00$ \\
\hline Ângulo $\left(^{\circ}\right)$ & $91,2 \pm 5,3$ & $83,3 \pm 8,9$ & $83,2 \pm 5,0$ & $84,2 \pm 5,0$ & $87,3 \pm 3,2$ & $89,0 \pm 5,6$ \\
\hline
\end{tabular}

Legenda: CON - grupo controle; EX - grupo exercício; EX+CT - grupo exercício + crioterapia; * diferença significativa $(p<0,05)$ em comparação ao repouso. 
$\mathrm{Na}$ variável dor, os maiores valores encontrados pertenceram nas sessões exercício, no qual foram encontradas diferenças significativas em $24 \mathrm{~h}, 48 \mathrm{~h}, 72 \mathrm{~h}$ e 96 horas em relação ao repouso no grupo EX. No entanto, é interessante notar que no grupo $\mathrm{EX}+\mathrm{CT}$ houve diferenças significativas apenas nos dois primeiros momentos (24 e 48 horas). $\mathrm{O}$ grupo controle não apresentou diferenças significativas.

Em relação ao ângulo da articulação, os menores valores estiveram localizados nos grupos exercício, os quais demonstraram reduções significativas nos momentos de medidas 24, 48, 72 e 96 horas em relação ao repouso. No grupo $\mathrm{EX}+\mathrm{CT}$ e $\mathrm{CON}$ não foram encontradas diferenças significativas.

\section{DISCUSSÃO}

Sabe-se que após uma sessão de treinamento com cargas externas, fisiologicamente acontecem modificações estruturais no músculo esquelético, que irão responder de acordo com o tipo, intensidade e duração do exercício. Tais alterações provocam aumento nos valores dos marcadores de agressão tecidual na corrente sanguínea e nas propriedades mecânicas, tais como: concentração de CK, sensação de dor e amplitude do movimento ${ }^{1,11}$.

Neste sentido, observa-se que as técnicas crioterápicas mais acessíveis para população de maneira geral, para prevenir e tratar as alterações no músculo após o exercício, são as bolsas térmicas e a massagem direta com o gelo, por serem de fácil acesso e baixo custo $^{4,12}$. No entanto, é interessante levar em consideração que por meio desses métodos, não é possível determinar a temperatura em que se está aplicando o procedimento entre a área afetada e o agente terapêutico. Assim, o sujeito pode estar adicionando à pele temperaturas não controladas para o tratamento e agravamento de lesões.

Dessa forma, no presente estudo foi utilizado o tratamento crioterápico com imersão, com temperatura constante, devido ao influxo e efluxo da água na caixa de tratamento crioterápico (resfriamento por convecção). Assim, de maneira pioneira o protocolo estabeleceu uma temperatura controlada à $15^{\circ} \mathrm{C}$, com quatro sessões de trinta minutos (uma a cada 12 horas). Este tempo foi suficiente para reduzir a temperatura muscular, assim como os impulsos excitatórios, agindo na prevenção e tratamento da lesão muscular induzida pelo exercício de força excêntrico. Vale salientar que tempos superiores a 30 minutos, podem favorecer o processo de ulceração e paralisia de nervos superficiais que circundam a área em tratamento ${ }^{13}$.
Observa-se que após a realização do exercício de força excêntrico, o grupo EX que não utilizou a técnica crioterápica, apresentou elevações significativas nos valores de CK sanguínea nos momentos 24 e 48 horas após a sessão de exercício em relação ao repouso. Por outro lado, o grupo $\mathrm{EX}+\mathrm{CT}$, exibiu incrementos apenas no período 24 horas, apresentando assim, a maior eficiência no tratamento da lesão celular induzida pelo exercício nesta variável. O resultado deste estudo foi encontrado de maneira similar por Baylei et al. ${ }^{10}$, que realizaram a técnica de imersão à $10^{\circ} \mathrm{C}$ durante 10 minutos, e encontraram alterações significativas nos níveis de CK entre 1 hora e 24 horas pós exercício, retornando aos valores iniciais 48 horas após a indução. Já no estudo de Vieira et al. ${ }^{14}$, ao comparar diferentes temperaturas $\left(5^{\circ} \mathrm{e} 15^{\circ} \mathrm{C}\right)$ de crioterapia com imersão durante 20 minutos imediatamente após uma sessão de exercício excêntrico, identificaram que a temperatura elevada gerou melhor perfil de recuperação, apresentando valores próximos ao de repouso no momento 72 horas pós exercício. Essa diminuição nos valores de $\mathrm{CK}$, pode ser proporcionada devido ao efeito da refrigeração constante no músculo, causada pela crioterapia moderada $\left(15^{\circ} \mathrm{C}\right)$, modulando a resposta inflamatória ${ }^{15,16}$.

No entanto, verifica-se na literatura que altos valores de CK estão intimamente associados com aumento da percepção de dor entre 24 e 48 horas após o exercício ${ }^{10,17,18}$. No presente estudo, esse fenômeno também ocorreu após a sessão de exercício de força excêntrico. Observou-se que o grupo sem a utilização da crioterapia, apresentou altos níveis de $\mathrm{CK}$, e permaneceu com valores de percepção subjetiva de dor significativamente maiores em relação ao repouso, verificando diminuição apenas após uma semana de realização da sessão. Resultado diferente foi encontrado no grupo $\mathrm{EX}+\mathrm{CT}$, que após 72 horas da sessão mostrou que os valores de percepção subjetiva de dor retornaram aos encontrados no repouso, demonstrando a eficiência da crioterapia na redução da percepção de dor. Essa reação pode ter ocorrido em resposta à vasoconstrição, a qual proporciona diminuição do fluxo sanguíneo e edema local, consequentemente favorece na redução dos níveis de $\mathrm{CK}$, bem como na desaceleração da ação metabólica celular ${ }^{4,6}$.

Em relação a amplitude de movimento, é possível notar de acordo com a Tabela 1, que apenas no grupo EX ocorreu diminuição significativa nos momentos 24,48 , 72 e 96 horas em relação ao momento repouso. Por outro lado, pode-se notar que o grupo $\mathrm{EX}+\mathrm{CT}$, não apresentou diferenças significativas em nenhum momento, mantendo os valores normais, apresentando assim, comportamento similar ao encontrado no $\mathrm{GC}$, o qual não realizou intervenção com exercício. 
De maneira contrária, no estudo de Isabell et al. ${ }^{19}$, com 22 sujeitos saudáveis $(20,3 \pm 2,1$ anos), ao utilizar a técnica com bolsas de gelo (temperatura não controlada), não encontraram resultados eficientes na manutenção do movimento e na inflamação muscular nos primeiros momentos (24 e 48 horas) após exercício, apresentando início de melhora nas variáveis analisadas apenas a partir de 72 horas. Com metodologia crioterápica divergente ao presente estudo, Howatson et al. ${ }^{15}$ ao realizar a aplicação do gelo e massagem diretamente no local lesionado após o exercício, não demonstraram resultados eficientes na redução da $\mathrm{CK}$, da manutenção de movimento e na sensação da dor em 12 adultos (24,8 $\pm 5,3$ anos). Reportando apenas pequena diminuição da inflamação muscular após um período de 72 horas. Por outro lado, no estudo realizado por Macedo et al. ${ }^{16}$, identificou-se que após a aplicação da crioterapia de imersão em água fria, houve diminuição significativa na atividade eletromiográfica da musculatura do membro inferior, apresentando eficiência no decréscimo da resposta inflamatória no músculo esquelético.

\section{REFERÊNCIAS}

1. Silva FOC, Macedo DV. Exercício físico, processo inflamatório e adaptação: uma visão geral. Rev Bras Cineantropom Desempenho Hum. 2011;13(4):320-8. doi: 10.5007/1980-0037.2011v13n4p320.

2. Berton RPB, Libardi CA, Conceição MS, Bonganha V, Nogueira FRD, Chacon-Mikahil MPT, et al. Dano muscular : resposta in fl amatória sistêmica após ações excêntricas máximas. Rev Bras Educ Fis Esporte. 2012;26(3):367-74. Disponível em: www.scielo.br/pdf/rbefe/v26n3/02.pdf

3. Duarte JA, Mota MP, Neuparth MJ, Appell HJ, Soares JMC. Miopatia do exercício. Anatomopatologia e fisiopatologia. Rev Port Ciên Desporto. 2001;1(2):73-80. Disponível em: www. fade.up.pt/rpcd/_arquivo/artigos_soltos/vol.1_nr.2/09.pdf.

4. Merrick MA, Rankin JM, Andres FA, Channing L. Hinman. A preliminary examination of cryotherapy and secondary injury in skeletal muscle. Med Sci Sport Exerc. 1999;31:1516-21. doi: 10.1097/00005768-199911000-00004.

5. Bleakley CM, Davison GW. Cryotherapy and inflammation: evidence beyond the cardinal signs. Phys Ther Rev. 2010;15(6):430-5. doi: 10.1179/1743288X10Y.0000000014.

6. Knight KL. Crioterapia no tratamento das lesões esportivas. São Paulo: Manole; 2000.

7. Freitas C, Luzardo R. Crioterapia: efeitos sobre as lesões musculares. Rev Episteme Transversalis. 2013;4(1).
Assim, de acordo com os resultados do presente estudo, a crioterapia de imersão pode ser utilizada como um tratamento eficaz na redução da miopatia do exercício e da DMT, pois proporciona redução na concentração da CK, e gera manutenção da amplitude do movimento de forma aguda. Contudo, uma possível limitação do estudo foi a utilização de uma amostra específica de adultos jovens, por isso os resultados não podem ser extrapolados para o público em geral. Necessitando a realização de estudos futuros, com outras populações.

\section{CONCLUSÃO}

Conclui-se que quatro sessões de trinta minutos (uma a cada 12 horas) de crioterapia moderada com imersão à $15^{\circ} \mathrm{C}$, mostrou-se uma alternativa viável para utilização clínica no processo de tratamento da miopatia do exercício e da DMT. Efeito demonstrado pela redução da CK, da dor sem palpação e pela recuperação ou manutenção da amplitude de movimento.

Disponível em: https://pdfs.semanticscholar.org/8744/ f829f7631c02f71d3400bf6d7603bba0b21a.pdf.

8. Santos T. Quantificação do uso da crioterapia no desempenho físico e tratamento de lesões na base de dados scielo.org. Rev Bras Prescrição Fisiol Exerc. 2014;8:44-9. Disponível em: www.rbpfex.com.br/index.php/rbpfex/article/view/582/544.

9. Pastre CM, Bastos FN, Neto Júnior J, Vanderlei LCM, Hoshi RA. Métodos de recuperação pós-exercício : uma revisão sistemática. Soc Bras Med Esporte. 2009;15:138-44. http:// dx.doi.org/10.1590/S1517-86922009000200012.

10. Clarkson PM, Hubal MJ. Exercise-induced muscle damage in humans. Am J Phys Med Rehabil. 2002;81(11):52-69. doi: 10.1097/01.PHM.0000029772.45258.43.

11. Barroso R, Roschel H, Gil S, Ugrinowitsch C, Tricoli V. Efeito do Número e intensidade das ações excêntricas nos indicadores de dano muscular. Rev Bras Med Esporte. 2011;17(6):401-4. http://dx.doi.org/10.1590/S151786922011000600006 .

12. Rupp KA, Herman DC, Hertel J, Saliba SA. Intramuscular temperature changes during and after 2 different cryotherapy interventions in healthy individuals. J Orthop Sports Phys Ther. 2012;42(8):731-7. doi: 10.2519/jospt.2012.4200.

13. Felice TD, Santana LR. Recursos fisioterapêuticos (crioterapia e termoterapia) na espasticidade: revisão de 
literatura. Rev Neurociên. 2009;17(1):57-62. Disponível em: www.luzimarteixeira.com.br/wp-content/uploads/2015/07/ CRIOTERAPIA-NA-ESPASTICIDADE.pdf

14. Vieira A, Siqueira AF, Ferreira-Junior JB, Carmo J, Durigan JLQ, Blazevich A, et al. The Effect of Water temperature during cold-water immersion on recovery from exerciseinduced muscle damage. Int J Sports Med. 2016;37:937-43. doi: $10.1055 / \mathrm{s}-0042-111438$

15. Howatson G, Gaze D, Van Someren KA. The efficacy of ice massage in the treatment of exercise-induced muscle damage. Scand J Med Sci Sports. 2005;15(6):416-22. doi: 10.1111/j.1600-0838.2005.00437.x

16. Macedo CSG, Alonso CS, Liporaci RF, Vieira F, Guirro RRJ. Cold water immersion of the ankle decreases neuromuscular response of lower limb after inversion movement. Braz J Phys Ther. 2014;18(1):93-7. doi: 10.1590/S141335552012005000132 .

Recebido em: 07.01.2015

Aceito em: 04.08.2017
17. Nosaka K, Newton M, Sacco P, Chapman D, Lavender A. Partial protection against muscle demage by eccentric actions at short muscle lengths. Med Sci Sports Exerc. 2005;37(5):746-53. doi: 10.1249/01. MSS.0000162691.66162.00.

18. Júnior EA, Brito CJ, Santos WOC, Valido CN, Mendes EL, Franchini E. Influence of cryotherapy on muscle demage marker in jiu-jitsu fighters after competition: a cross-over study. Rev Andaluza Med Deport. 2014;7(1):7-12. https:// doi.org/10.1016/S1888-7546(14)70054-1.

19. Isabell WK, Durrant E, Myrer W, Anderson S. The effects of ice massage, ice massage with exercise, and exercise on the prevention and treatment of delayed onset muscle soreness. J Athl Train. 1992;27(3):208-17. Available from: https:// www.ncbi.nlm.nih.gov/pmc/articles/PMC1317248/pdf/ jathtrain00035-0018.pdf. 